\section{Cursos Cortos de Perfeccionamiento y Especialización en Lima Callao para los Trabajadores de Construcción Civil}

\author{
Short Courses of Perfection and Specialization in Lima Callao \\ for Civil Construction Workers
}

\begin{abstract}
RESUMEN
Según el Ministerio de Educación, son diversas las instituciones que brindan y desarrollan formas de educación y capacitación que posibilitan el acceso a la formación técnica para la población dispersa o con dificultad para asistir a centros que brindan especializaciones a altos costos, este es el caso de las instituciones técnicas regentadas por el Estado. Como estrategia inicial, se planteó la realización de una sistematización de experiencias en educación técnica en construcción en Sudamérica, específicamente en los cursos de perfeccionamiento y especialización de técnicos enfocados al mercado de la construcción, que sirvan de marco a futuras propuestas de educación técnica eficiente y eficaz, valorar y reconocer la importancia de las propuestas formativas en educación técnica y formación laboral complementaria.
\end{abstract}

Palabras claves: Programas de perfeccionamiento; especialización técnica; formación laboral; construcción civil.

\begin{abstract}
According to the Ministry of Education, there are several institutions that provide and develop forms of education and training that allow access to technical training for the dispersed population or with difficulty to attend centers that offer specializations at high costs, this is the case of the technical institutions run by the State. As an initial strategy, the realization of a systematization of experiences in technical education in construction in South America, specifically in the courses of improvement and specialization of technicians focused on the construction market, that serve as a framework for future proposals of efficient technical education and effective, assess and recognize the importance of training proposals in technical education and complementary job training.

Keywords: Improvement programs; technical specialization; job training; civil construction.
\end{abstract}

\section{Luzmila Olinda Matos Cubas}

luzmilamatos@gmail.com

Servicio Nacional de Capacitación para la Industria de la Construcción (SENCICO) 


\section{INTRODUCCIÓN}

El presente artículo tiene como objetivo reconocer a la educación como eje principal del desarrollo de las capacidades y potencialidades de las personas incluidas en el sector construcción, de manera que dotadas de capacidades laborales se integren en la comunidad de modo participativo, crítico y productivo.

El Portal del Servicio Nacional de Capacitación para la Industria de la Construcción (SENCICO, 2019) describe que en Perú existen diversas instituciones encargadas de la educación y capacitación técnica especializada, como SENCICO, que fue creada por Decreto Ley 21673 - Ley Orgánica del Servicio Nacional de Capacitación para la Industria de la Construcción. Institucionalmente inicia el desarrollo de sus funciones en octubre de 1976 instalándose el Primer Consejo Directivo Nacional a cargo del Ing. Jorge Cáceres y sus operaciones en 1977. Ya para 1978 expande sus centros de capacitación al interior del país (Ica, Cusco, Huánuco, Tacna y otros). En 1981 se modificó la Ley Orgánica que lo regía bajo el Decreto Ley 21673 y entra en vigencia la Ley 147, vigente hasta la actualidad. Es importante considerar que económicamente se financia principalmente con el aporte de personas naturales y jurídicas que perciben ingresos por contrataciones de obras de construcción civil.

Acerca de la organización en SENCICO, jerárquicamente es el Consejo Directivo Nacional representado por la Presidencia Ejecutiva quien encabeza la institución y es el ente encargado de la conducción y dirección de acuerdo a las políticas vigentes establecidas, se desglosa seguidamente en la Gerencia General encargado de dirigir, coordinar y controlar las actividades administrativas, de investigación de normalización y académicas dentro de la institución, ambas en el aspecto administrativo. Enfocarse en el aspecto académico permite mencionar a la Gerencia de Formación Profesional y las Gerencias Zonales que cumplen el rol de áreas normativas y ejecutoras.

SENCICO es un centro especializado en la educación técnica para la industria de la construcción con su Programa de Formación de Profesionales Técnicos, como medio, a través del que se logra la educación de técnicos, con carreras de cuatro a seis semestres académicos, brindando educación no universitaria de nivel superior y capacitando con herramientas tecnológicas de vanguardia para lograr competitividad internacional en su especialidad. En el ámbito de la capacitación técnica el boom inmobiliario (2006) hizo que se requiriera profesionales capacitados debido al incremento de los avances tecnológicos, globalización económica y cambio permanente de los escenarios laborales, siendo de relevancia las carreras técnicas que se realizan en las Sedes de las Escuela Superior Técnica SENCICO (EST), ya que permiten la diversidad de la formación técnica que requieren las empresas innovadoras a partir de características del sector construcción.

SENCICO se encarga además de capacitar en programas de cursos modulares o cursos cortos de habilitación, que permiten brindar capacitación y perfeccionamiento a los estudiantes y/o profesionales que se adhieren a este tipo de actividad educativa, tales como el Programa de Formación de Técnicos Operativos, Programa de Formación de Operativos y Programa de Perfeccionamiento y Especialización. Es importante la infraestructura y equipos actualizados, que facilitan la formación de nuevos conocimientos con una educación integral, inmersos dentro de las exigencias del mercado laboral de hoy.

En los últimos años, la capacitación a través de cursos cortos en SENCICO se ha ido incrementando, lo que permite darnos una idea de la capacidad de soporte de capacitación que presenta en los últimos 3 años, a través de las siguientes cifras:

\section{Tabla 1}

Total de Capacitados en Lima - Callao-Años 2016 - 2018

\begin{tabular}{|c|c|}
\hline \multicolumn{2}{|c|}{ Total de Capacitados Lima Callao (por año) } \\
\hline 2016 & 14,577 \\
\hline 2017 & 20,692 \\
\hline 2018 & 25,698 \\
\hline
\end{tabular}

Fuente: Elaboración propia. Basado en Memoria Anual SENCICO 2016 - 2017 - 2018

\section{MÉTODOS}

Se usó el método de tipo deductivo, puesto que el presente estudio va de lo general a lo específico. Esto se sustenta en lo afirmado por Dávila 


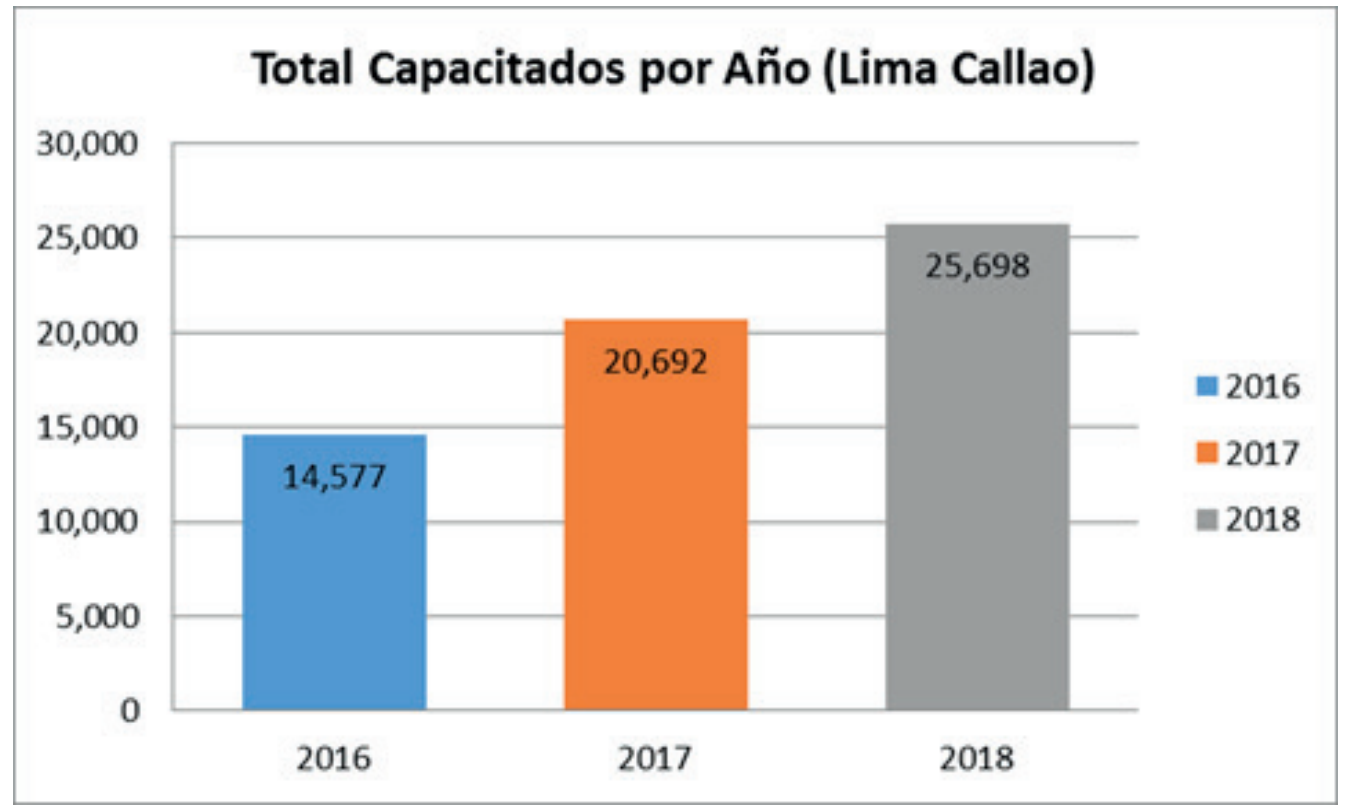

Figura 1. Total de Capacitados en Lima - Callao-Años 2016 - 2018. Elaboración propia. Basado en Memoria Anual SENCICO 2016 - 2017 - 2018

(2006) que de manera exacta indica que "la deducción permite establecer un vínculo de unión entre teoría y observación y permite deducir a partir de la teoría los fenómenos objeto de observación" (p. 181).

Entonces, la deducción implica el paso de los resultados obtenidos al planteamiento de hipótesis, leyes y teorías que engloban los casos de los que se partió generalizando resultados de manera mecánica, para buscar la comprensión de síntesis racionales.

\section{RESULTADOS}

Vásquez (2019) afirma que en el Perú existe ausencia de personal técnico calificado y este resulta en un problema que afecta al empresariado peruano. El autor reconoce que las instituciones de educación técnica deben informar y actualizar sus planes de estudio en pro de afianzar la confianza y seguridad de estudiar con docentes preparados, con temas y evaluaciones que se centren en competencias concretas buscando educación no sólo teórica, sino también práctica.

Ploog (2018) como Presidente del Gremio de Importadores de Maquinarias, Equipos, Repuestos y Herramientas de la CCL afirma que existe un déficit elevado de trabajadores técnicos y reconoce que la oferta educativa técnica para determinadas especialidades adolece de conocimiento práctico. Ploog considera importante realizar visitas a las empresas nacionales a fin de hacer comparaciones entre lo que se enseña y lo que se requiere en el campo, difundiendo información acerca de las capacidades y formación que pueden recibir en las instituciones de capacitación técnica a través de cursos cortos.

Para González (2018), Economista y Ex Ministro de Trabajo, el principal problema del país en el tema de empleo técnico es el nivel de calidad y cantidad que ofrecen las instituciones de capacitación, considera que es mayor la cantidad de personal técnico que requieren las empresas en la actualidad y existe gran déficit educativo, afirma además que son pocas las instituciones públicas o privadas que ofrecen servicios de capacitación especializada, y que son las empresas quienes muchas veces se encargan de cubrir ese costo para su personal.

En la Memoria Anual SENCICO 2017, Estrada (ex Presidente Ejecutivo de SENCICO) reconoce que el sector construcción es uno de los que más requiere de técnicos capacitados, ya que según estadísticas del Ministerio de trabajo existe 460 mil trabajadores que rotan en ese sector y hay más de un millón de personas relacionadas al negocio de la construcción de 
manera empírica con conocimientos prácticos adquiridos sin la capacitación ý especialización técnica requerida. SENCICO es la única institución pública de capacitación presente en 19 ciudades en todo el Perú, que busca tecnificar a quienes trabajen de manera empírica a través de capacitaciones masivas con gobiernos locales o empresas agrupadas en pro de cursos adecuados en albañilería, instalaciones eléctricas y sanitarias.

SENCICO a través de Convenios de Cooperación Institucional con entidades públicas y privadas consigue fomentar, perfeccionar y fortalecer los programas de formación - capacitación tanto en territorio local, regional, nacional e internacional; sea esta cooperación suscribiendo Convenios Marco y Convenios Específicos. Cabe mencionar que los convenios son ejecutados por las Gerencias Zonales, en Lima Callao SENCICO ha suscrito convenios de capacitación en cursos cortos con empresas privadas tales como: Comité Nacional de Administración el Fondo para la Construcción de Viviendas y Centros Recreacionales para los Trabajadores en Construcción Civil en el Perú (CONAFOVICER), Confederación de Trabajadores CTP, la Federación de Trabajadores de Construcción Civil y de Actividades Afines del Perú (FETRACCAAP), Federación de trabajadores en construcción del Perú (FTCCP), Federación Unitaria de Trabajadores en Construcción Civil y de Actividades Similares del Perú (FUTCCASP), Graña y Montero, Perú UP, Fundación Suiza de Cooperación para el Desarrollo Técnico (SWISSCONTACT); y con instituciones públicas como el MEM-CARELEC (Consejo de Administración de Recursos para la Capacitación de Electricidad), Organismo de Formalización de la Propiedad Informal (COFOPRI), Ministerio de Defensa, Ministerio de Justicia y Derechos Humanos y el Servicio Nacional de Agua Potable y Alcantarillado en Lima (SEDAPAL).

De otro lado, se considera a CAPECO (Cámara Peruana de Construcción) como institución particular encargada de ofrecer programas educativos para profesionales técnicos en construcción civil, capacitando en el uso de herramientas informáticas y tecnológicas para la construcción, brindando a la sociedad personal capacitado bien cotizado en el mercado laboral, dictando cursos como: instalaciones eléctricas, lectura de planos, metrados y presupuestos, supervisión de obras, seguridad y salud en la construcción, entre otros. (Portal CAPECO, 2019)

A nivel Sudamérica sobre centros de especialización se tiene la propuesta de creación del Instituto de Capacitación para el Obrero de la Construcción en Chile de parte de Fuenzalida (2010), que englobaría instituciones como la Universidad Tecnológica de Chile (INACAP), Capacita SA, la Cámara Chilena de Construcción (CCHC), Corporacion Sigdo Koppers (SK) Capacitación, Centro Técnico INDURA entre otros, que dictan cursos certificados por el SENCE (Servicio Nacional de Capacitación y Empleo - Chile) como tecnología de la construcción, instalaciones sanitarias, albañilería y estuco, entre otros; considerando como relevante y mejor opción en el rubro de construcción a la Institución Gremial de la Cámara Chilena de la Construcción (CCHC).

En Argentina se halla a CAMARCO (Cámara Argentina de Construcción) que constituye el mayor gremio de empresarios del sector de la construcción con proyección federal y que se encarga de los procesos de intercambio y debate de los actores de la industria. CAMARCO, forma parte de la Federación Interamericana de la Industria de la Construcción (FIIC) y la Asociación de Confederaciones Internacionales de Construcciones (CICA) instituciones que buscan instituir lineamientos específicos en el sector construcción.

En el aspecto teórico se plasma la definición de conceptos importantes como el de perfeccionamiento, "proceso de formación especializada y de corta duración que suele impartirse tras la educación o formación inicial, y cuyo objetivo consiste en complementar, mejorar o actualizar las destrezas, las competencias y los conocimientos adquiridos con la formación previa" (Centro Europea para el Desarrollo de la Formación Profesional, CEDEFOP, 2004).

Y para la definición especialización, citamos a Borisov, Zhamin y Makarova (2009) como una manera de división social de trabajo entre distintas ramas de la industria y la producción, la especialización presenta carácter planificado en determinadas partes de procesos dentro de una industria específica enfocado hacia determinadas actividades económicas. 
Enfocarse en el estudio específico de SENCICO, se trata de dar a conocer la manera de capacitación de los Programas de cursos modulares o cursos cortos de habilitación que se desarrollan en las Gerencias Zonales del SENCICO, que se dirigen específicamente a trabajadores del sector construcción y público general (jóvenes y/o adultos) que deseen incorporarse a esta actividad para lograr sistemáticamente su calificación en una ocupación específica. Los cursos en este tipo de programas son terminales, de corta duración, y se desarrollarán en horarios de mañanas, tardes y/o noches en jornadas de tres (03) horas diarias como mínimo $\mathrm{y}$ un promedio de mes y medio por curso. Estos programas se ejecutan aplicando la normatividad técnico pedagógica que imparte la Gerencia de Formación Profesional del SENCICO.

El Programa de Calificación Ocupacional está estructurado de la forma siguiente:
La estructura es clara, muestra la organización por cursos que comprenden dos áreas: técnica (por módulos o cursos cortos) y social (Desarrollo Laboral), con la finalidad de darle integridad a la capacitación.

\section{Modalidades de la capacitación en SENCICO.}

Se cuenta con:

- Capacitación en Centro de Formación, Modalidad mediante la cual la capacitación se desarrolla en Aulas, Talleres y en Laboratorios, efectuándose las prácticas simuladas mediante tareas pedagógicas.

- Capacitación en la Empresa (en obra) y Capacitación Producción, Modalidad en la que las actividades educativas técnico productivas de carácter formativo, se desarrollan mediante la suscripción de convenios con empresas del Sector, en cursos

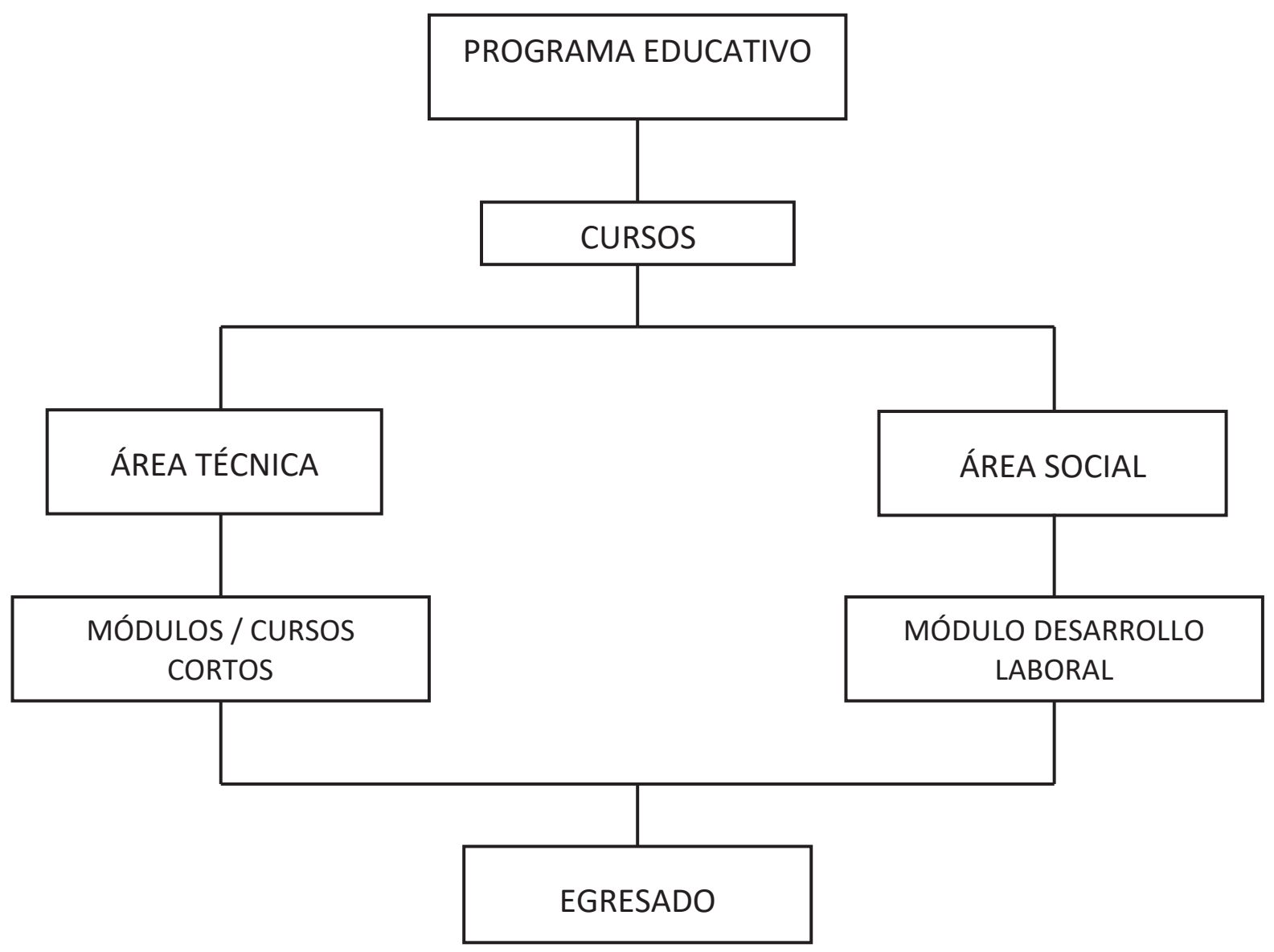

Figura 2. Organigrama del Programa de Calificación Ocupacional. Fuente: SENCICO: Gerencia de Formación Profesional (2009, p. 45) 
de capacitación específica que se determina en el Plan de Capacitación pertinente, para obtener conocimientos teóricos prácticos mediante el aprendizaje de las tareas convenientes.

Sobre el sistema de capacitación se utiliza el sistema modular, que es una de las mejores estrategias para lograr la capacitación de los trabajadores que requiere el mercado laboral, porque orgánicamente, permite cambios en función de las aspiraciones y posibilidades de trabajo de los individuos que participan en él, ofreciéndoles oportunidades de "entradas y salidas", en el proceso, durante su vida formativa, de acuerdo a sus necesidades y sus características individuales.

La Implementación de la infraestructura educativa, que se basa en la programación educativa aprobada para cada órgano de ejecución, el Especialista Educativo de la Zonal, se encarga de lo necesario para la ejecución de los cursos (aula, taller, herramientas, materiales, equipo, hojas de instrucción, etc.), todo esto, de acuerdo a los contenidos teórico - prácticos de los programas curriculares. Esto es realizado antes del inicio de los cursos.

Acerca de la Selección y contratación de docentes, son los órganos de ejecución quienes seleccionan a los docentes que desarrollan los cursos comprendidos en todos los programas de la Zonal, a través de la relación de banco de expositores previamente aprobada por la Gerencia de Formación Profesional.

Acerca de la difusión y promoción de los cursos, esto corresponde al Especialista de Promoción Educativa, quien establece y conduce los procedimientos de promoción y convocatoria de los cursos en forma oportuna y efectiva, según la programación dada por cada Zonal.

Se debe mencionar el proceso de desarrollo curricular, que se inicia con un curso o módulo, presentado por las autoridades de la Zonal, siendo de responsabilidad del Especialista Educativo. En este acto se presenta al docente o docentes, se indica la estructura del curso, requisitos de asistencia, reglamento del participante y la línea de autoridad para recibir inquietudes de los participantes; así como otras disposiciones específicas. Se efectúan evalua- ciones que permiten diagnosticar las habilidades y conocimientos de los participantes.

La fase de desarrollo de los contenidos, de responsabilidad del docente, se hace de acuerdo a la estrategia metodológica para la capacitación de los programas educativos siguiendo la programación analítica. El planeamiento de cada clase o sesión de aprendizaje se indica en el Programa Analítico y se aplica en el Plan de Actuación. El transcurso del desarrollo de los contenidos busca que el especialista educativo supervise y observe, valorando, asesorando y corrigiendo la labor del docente y comportamiento de los participantes.

Acerca de algunas recomendaciones para el desarrollo curricular, se tiene en cuenta cubrir todos los contenidos curriculares a partir de las capacidades de cada Unidad de Competencia.

Es importante reconocer la importancia del Programa de Perfeccionamiento y Especialización en cursos de alta rotación como: lectura de planos, trazado y replanteo de obra, reparación y mantenimiento de instalaciones, encofrado metálico, metrado y estimación de costos, manejo de equipos topográficos: estación total y GPS, entre otros.

\section{Alto nivel de rotación en cursos desarro- llados por la Zonal Lima Callao.}

Entre los cursos que se dictan frecuentemente, tenemos los de lectura de planos que se caracterizan en:

- Lectura de planos de arquitectura, curso en el que se adquiere conocimientos sobre la interpretación de planos de cortes, detalles, localización, ubicación, simbología y escalas, elevaciones, plantas y seguridad en proyectos de viviendas y edificios comerciales. Duración 25 horas.

- Lectura de planos de estructuras, en el que se adquiere conocimientos sobre la interpretación de planos de detalles de columnas, muros de concreto, cimentación, encofrados de contra pisos y techos, detalle de cisterna y tanque elevado, simbología y especificaciones técnicas en proyectos de estructuras en viviendas y/o edificación comercial. Duración 24 horas. 
- Lectura de planos de instalaciones eléctricas, en el que se adquiere la habilidad de analizar e interpretar los planos de redes de alumbrado y salidas de fuerza, redes de tomacorrientes, detalles de diagramas de montantes, unifilares, simbologías y especificaciones técnicas de los proyectos de instalaciones eléctricas en viviendas y edificios multifamiliares. Duración 24 horas.

- Lectura de planos de instalaciones sanitarias, en el que el estudiante se capacita en el análisis de planos de redes de agua fría y caliente, redes de desagüe y ventilación, detalles de las isometrías y diagramas de montantes, cisterna, tanque elevado, tanque hidroneumático $\mathrm{y}$ calentador de agua y lectura general de los planos de proyectos de instalaciones sanitarias en viviendas y edificaciones multifamiliares. Duración 24 horas.

- Lectura de planos de instalaciones de gas, en la que se adquiere los fundamentos de la instalación de gas, el reconocimiento del suministro, acometida a medidor - regulador, detalles de la instalación de gas en edificios, planos isométricos, según especificaciones técnicas, normas de seguridad y calidad establecidas. Duración 24 horas.

Los resultados indican de manera porcentual lo siguientes:

\section{Tabla 2}

Nivel Operativo - Programa de Perfeccionamiento y Especialización -Cursos de Lectura de Planos (Enero - junio 2019)

\begin{tabular}{|c|l|c|c|}
\hline № & Nombre del curso & Cant. Participantes & \% \\
\hline 1 & Arquitectura & 738 & 30 \\
\hline 2 & Estructuras & 621 & 25 \\
\hline 3 & Instalaciones de Gas & 123 & 5 \\
\hline 4 & Instalaciones Eléctricas & 504 & 21 \\
\hline 5 & Instalaciones Sanitarias & 456 & 19 \\
\hline & TOTAL & 2442 & 100 \\
\hline
\end{tabular}

Fuente: Elaboración propia. Basada en información de Centros de Formación - SENCICO LIMA CALLAO

Queda marca la tendencia de alta rotación en los cursos de especialización de arquitectura y estructuras.

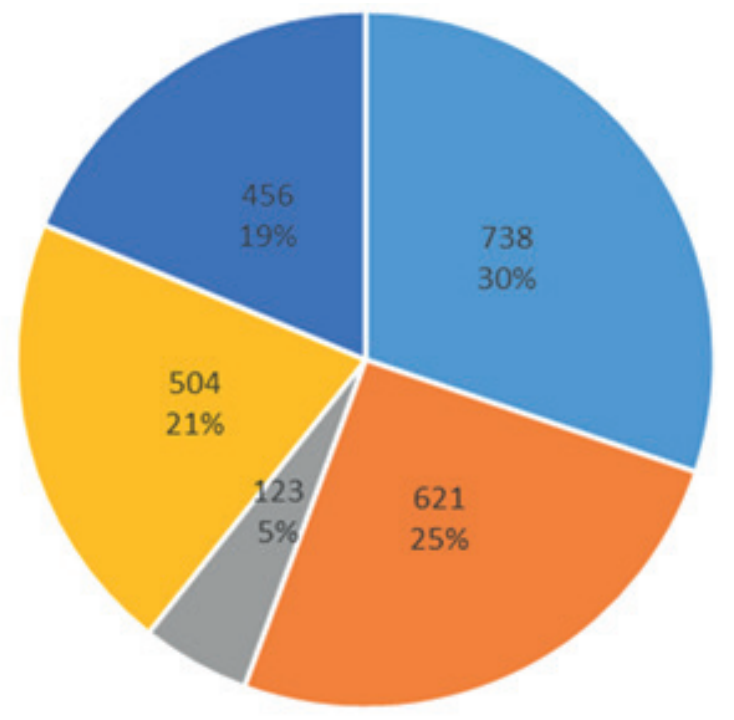

- Arquitectura

- Estructuras

- Instalaciones de Gas

= Instalaciones Electricas

- Instalaciones Sanitarias

Figura 3. Nivel Operativo - Programa de Perfeccionamiento y Especialización -Cursos de Lectura de Planos (Enero - junio 2019). Elaboración propia. Basada en información de Centros de Formación - SENCICO LIMA CALLAO

\section{DISCUSIÓN}

Respondiendo a la necesidad del mercado, SENCICO se ha mostrado como una institución capaz de cubrir las necesidades de capacitación que requieren las empresas en el sector construcción, brindando cursos de especialización específica en los que se adquiere los conocimientos requeridos. Al ser SENCICO una institución pública que depende del Ministerio de Vivienda, Construcción y Saneamiento, de quien se desprende la realidad en el sector a través de las estadísticas que muestran la carencia de personal especializado capaz de ser incluido en el mercado laboral del rubro, pero a la vez se debe considerar los lineamientos del Ministerio de Educación, como ente encargado de proponer las estrategias educativas y de enseñanza que deben ser adquiridas por las personas que requieran o deseen ser capacitados en SENCICO. 
En el aspecto económico se debe tener en cuenta de una parte, que SENCICO maneja y gestiona sus costos contando con el aporte que recibe de parte de las empresas constructoras, que aportan el 2 por mil ( $2 \%$ ) de sus ingresos para soportar los cursos y otras necesidades de la institución. De otro lado, los participantes en los cursos pagan una tarifa mínima que es aprobada por la Alta Dirección. La captación de los ingresos de cursos es gestionada por la Oficina de Administración y Finanzas y el Departamento de Tesorería dentro de la normatividad vigente.

Se reconoce acerca de la planificación estratégica, las diferencias que marcan ser una institución del Estado (SENCICO) y ser una institución privada (CAPECO), las estrategias de mejora y capacitación son marcadamente diversas, por un lado, se depende específicamente de la normatividad y manejo que asigne el Estado para la institución, mientras que CAPECO maneja, propone y mejora sus políticas de capacitación, innovación, manejo de estrategias y ejecución.

De otro lado, no se encontró antecedentes que reconozcan estadísticamente la necesidad de implementación de más y mejores cursos al alcance de quienes requieran conocimiento que les permita adherirse al mercado de la construcción y poseer las capacidades y conocimientos requeridos en el manejo de nuevas actividades en un mercado creciente.

SENCICO como institución educativa busca en todo momento, responder a los requerimientos, tanto profesionales como de capacitación específica, entre sus estudiantes, requerimientos que son recogidos de manera directa a través de comunicación fluida con las empresas (desde hace más de cuatro décadas en promedio), las mismas que buscan ser cubiertas en los cursos de perfeccionamiento y especialización dictados por SENCICO.

Finalmente, la discusión se cierra con la comparación de instituciones con el mismo perfil en Sudamérica, por ejemplo, en la realidad chilena, en la que se tiene a Universidad Tecnológica de Chile INACAP, Capacita SA, CCHC, SK Capacitación, Centro Técnico INDURA manejados por el Servicio Nacional de Capacitación y Empleo de Chile, que se encar- ga de mejorar el aprendizaje y adquisición de conocimiento de las personas en el rubro de construcción. Tal cual la realidad de CAPECO en el Perú, Argentina cuenta con CAMARCO que engloba al gremio de empresarios de la construcción con la finalidad de instruir en lineamientos específicos del sector. Perú no se queda atrás en la implementación de nuevas políticas de mejora tanto de parte del sector público o Estado (SENCICO) y como de parte de la Inversión privada (CAPECO), ambas instituciones ofrecen capacitación especializada en diversas actividades.

\section{CONCLUSIONES}

Existen en el Perú diversas instituciones que brindan formas de educación y capacitación que posibilitan el acceso a la formación técnica y especializaciones específicas ante las necesidades del mercado de la construcción como un mercado emergente. Perú, cuenta con diversas instituciones encargadas de la educación y capacitación técnica especializada, como SENCICO y CAPECO.

SENCICO se reconoce como un centro especializado en la educación técnica para la industria de la construcción y cuenca con Escuelas Superiores Técnicas (EST) que brindan educación técnica especializada a través de herramientas tecnológicas de vanguardia.

Existe en la realidad peruana ausencia de personal técnico calificado, resultando un problema que afecta al empresariado peruano. Se debe dar realce e importancia a la contratación de profesionales con capacidades concretas buscando educación no sólo teórica, sino también práctica.

SENCICO depende del Ministerio de Vivienda en la parte de necesidades en la formación, pero a la vez considera los lineamientos del Ministerio de Educación, como ente encargado de proponer las estrategias educativas y de enseñanza, mientras que en el aspecto económico y de financiamiento se depende del Ministerio de Economía y Finanzas que se encargan de asignar los recursos.

En Sudamérica se han replicado experiencias como las de SENCICO y CAPECO en el Perú, en Chile se cuenta con la Universidad Tecnoló- 
gica de Chile INACAP, Capacita SA, CCHC, SK Capacitación, Centro Técnico INDURA manejados por el Servicio Nacional de Capacitación y Empleo de Chile y en Argentina se cuenta con CAMARCO que engloba al gremio de empresarios de la construcción con la finalidad de instruir en lineamientos específicos del sector.

\section{REFERENCIAS BIBLIOGRÁFICAS}

Dávila, G. (2006) El razonamiento inductivo y deductivo dentro del proceso investigativo en ciencias experimentales y sociales. Revista Laurus, vol. 12, núm. Ext, 2006, pp. 180-205 Universidad Pedagógica Experimental Libertador Caracas, Venezuela.
González J. (2018). Entrevista Diario Gestión. Economista y Ex Ministro de Trabajo. En: https:// gestion.pe/noticias/jorge-gonzalez-izquierdo/

Ploog (2018). Entrevista Diario Gestión. Presidente del Gremio de Importadores de Maquinarias, Equipos, Repuestos y Herramientas de la CCL. En: https://gestion.pe/impresa/ importacion-maquinarias-equipos-crecera-5-45370-noticia/

Portal web. SENCICO (2019). Recuperado de: https://www.sencico.gob.pe/publicaciones. php?id=127Fuenzalida G. (2010). Instituto de Capacitación para el Obrero de la Construcción y su entorno familiar y comunitario. Universidad de Chile. En: http://www.tesis.uchile.cl/ tesis/uchile/2010/aq-fuenzalida_g/pdfAmont/ aq-fuenzalida_g.pdf 
